PROCEEDINGS OF THE

AMERICAN MATHEMATICAL SOCIETY

Volume 30, Number 2, October 1971

\title{
A THEOREM ON MEAN-VALUE ITERATIONS
}

\author{
R. L. FRANKS AND R. P. MARZEC
}

Abstract. In this paper we consider a function which continuously maps a closed interval of the real line into itself. It is shown that a particular mean-value iterative scheme always converges to a fixed point. The result is known for functions which have a unique fixed point. This condition is not required here.

1. Introduction. In this paper we consider a function $f$ which continuously maps the closed interval $[0,1]$ into itself. We prove that a certain mean-value iterative scheme always converges to a fixed point of $f$ on $[0,1]$. This result was proved in [1], where $f$ was required to have a unique fixed point in the interval. In this paper we show that this restriction is unnecessary, convergence is proved by considering only the continuity of $f:[0,1] \rightarrow[0,1]$.

2. A convergent iterative scheme. Consider a function $f(x)$ with the following properties.

(i) $f(x)$ is continuous on $[0,1]$.

(ii) $f(x)$ maps $[0,1]$ into itself.

From Brouwer's fixed-point theorem, the function has at least one fixed point on this interval. We will now show that a particular mean-value iterative scheme converges to a fixed point.

THEOREM. Let $f(x)$ continuously map the closed interval $[0,1]$ into itself. Then the iterative scheme

$$
\begin{gathered}
x_{n+1}=f\left(\bar{x}_{n}\right), \\
\bar{x}_{n}=\sum_{i=1}^{n} x_{i} / n, \quad n=1,2,3, \cdots, \\
\bar{x}_{1}=x_{1} \in[0,1]
\end{gathered}
$$

converges to a fixed point of $f(x)$ on $[0,1]$.

Proof. We first state some properties which will be useful in later developments. Combining equations (1) and (2) gives

$$
\bar{x}_{n+1}=\frac{f\left(\bar{x}_{n}\right)-\bar{x}_{n}}{n+1}+\bar{x}_{n}, \quad n=1,2, \cdots .
$$

Received by the editors December 1, 1970.

AMS 1970 subject classifications. Primary 26A18; Secondary 40A05. 
Since both $\bar{x}_{n}$ and $f\left(\bar{x}_{n}\right)$ are in $[0,1]$, we obtain

$$
\left|\bar{x}_{n+1}-\bar{x}_{n}\right| \leqq \frac{1}{n+1}, \quad n=1,2, \cdots .
$$

This means the step size becomes arbitrarily small as $n$ increases. The proof can now be accomplished in two steps.

1. We first show that $\left\{\bar{x}_{n}\right\}$ converges. The sequence $\left\{\bar{x}_{n}\right\}$ is contained in $[0,1]$ so it has at least one limit point. For sake of contradiction assume $\xi_{1}$ and $\xi_{2}$ are two distinct limit points of $\left\{\bar{x}_{n}\right\}$ and $\xi_{1}<\xi_{2}$.

a. We will show that a consequence of this assumption is that $f(x)$ $=x$ for every $x$ in $\left(\xi_{1}, \xi_{2}\right)$.

Pick any $x^{*} \in\left(\xi_{1}, \xi_{2}\right)$. If $f\left(x^{*}\right)>x^{*}$, then by the continuity of $f$ there is a $\delta \in\left(0,\left(x^{*}-\xi_{1}\right) / 2\right)$ such that $f(x)>x$ for all $x$ satisfying $\left|x-x^{*}\right|$ $<\delta$. I.e.,

$$
\left|\bar{x}_{n}-x^{*}\right|<\delta \text { implies } \bar{x}_{n+1}>\bar{x}_{n} .
$$

Now (5) implies there is a number $N$ such that

$$
\left|\bar{x}_{n+1}-\bar{x}_{n}\right|<\delta, \quad n=N, N+1, \cdots .
$$

Also $\xi_{2}>x^{*}$ is a limit point of $\left\{\bar{x}_{n}\right\}$ so this $N$ can be chosen such that $\bar{x}_{N}>x^{*}$. But, by (6) and (7),

$$
\bar{x}_{n}>x^{*}-\delta>\xi_{1}, \quad n=N, N+1, \cdots,
$$

which means $\xi_{1}$ is not a limit point of $\left\{\bar{x}_{n}\right\}$, contrary to our assumption.

If $f\left(x^{*}\right)<x^{*}$, similar reasoning contradicts the assumption that $\xi_{2}$ is a limit point. Therefore $f\left(x^{*}\right)=x^{*}$ for all $x^{*} \in\left(\xi_{1}, \xi_{2}\right)$.

b. We will now show that $\xi_{1}$ and $\xi_{2}$ are not both limit points. Notice that

$$
\bar{x}_{n} \notin\left(\xi_{1}, \xi_{2}\right) \quad \text { for all } n=1,2, \cdots
$$

since if $f\left(\bar{x}_{n}\right)=\bar{x}_{n}$ then, by $(4), \bar{x}_{m}=\bar{x}_{n}$ for all $m>n$ and neither $\xi_{1}$ nor $\xi_{2}$ could be limit points.

Also (5) and (8) imply that there is a number $M$ such that if $\bar{x}_{M} \geqq \xi_{2}$ then $\bar{x}_{n} \geqq \xi_{2}>\xi_{1}$ for all $n>M$, and $\xi_{1}$ is not a limit point. If $\bar{x}_{M} \leqq \xi_{1}$ then $\bar{x}_{n}<\xi_{1}<\xi_{2}$ for all $n>M$ and $\xi_{2}$ is not a limit point. Either way, $\left\{\bar{x}_{n}\right\}$ cannot have two distinct limit points. Therefore $\left\{\bar{x}_{n}\right\}$ converges to its unique limit point, $\xi$.

2. To show that $f(\xi)=\xi$, assume $f(\xi)>\xi$. Let $\epsilon=(f(\xi)-\xi) / 2>0$. $\left\{\bar{x}_{n}\right\}$ converges to $\xi$ and $f$ is continuous so there is a number $N$ such 
that $f\left(\bar{x}_{n}\right)-\bar{x}_{n}>\epsilon$ for all $n>N$. By $(4)$,

$$
\bar{x}_{n+1}-\bar{x}_{n}=\frac{f\left(\bar{x}_{n}\right)-\bar{x}_{n}}{n+1}>\frac{\epsilon}{n+1} .
$$

Therefore

$$
\begin{aligned}
\lim _{m \rightarrow \infty}\left(\bar{x}_{N+m}-\bar{x}_{N}\right) & =\lim _{m \rightarrow \infty} \sum_{n=N}^{m-1}\left(\bar{x}_{n+1}-\bar{x}_{n}\right) \\
& \geqq \lim _{m \rightarrow \infty} \sum_{n=N}^{m-1} \frac{\epsilon}{n+1}=\infty .
\end{aligned}
$$

Therefore $\bar{x}_{n} \rightarrow \infty$, contradicting the fact that $\bar{x}_{m} \in[0,1]$ for all $m$.

Similarly, assuming $f(\xi)<\xi$ implies $\bar{x}_{n} \rightarrow-\infty$. Therefore $f(\xi)$ $=\xi$. Q.E.D.

\section{REFERENCES}

1. W. R. Mann, Mean value methods in iteration, Proc. Amer. Math. Soc. 4 (1953), 506-510. MR 14, 988.

Bell Telephone Laboratories, Whippany, New Jersey 07981 